\title{
A Maximum Radius for Habitable Planets
}

\author{
Yann Alibert ${ }^{1}$
}

Received: 17 June 2014 / Accepted: 28 July 2014 /

Published online: 10 July 2015

(C) Springer Science+Business Media Dordrecht 2015

\begin{abstract}
We compute the maximum radius a planet can have in order to fulfill two constraints that are likely necessary conditions for habitability: 1- surface temperature and pressure compatible with the existence of liquid water, and 2- no ice layer at the bottom of a putative global ocean, that would prevent the operation of the geologic carbon cycle to operate. We demonstrate that, above a given radius, these two constraints cannot be met: in the Super-Earth mass range (1-12 $\left.\mathrm{M}_{\text {earth }}\right)$, the overall maximum that a planet can have varies between 1.8 and

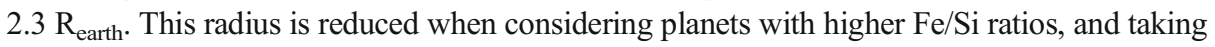
into account irradiation effects on the structure of the gas envelope.
\end{abstract}

Keywords Planet structure $\cdot$ Habitability $\cdot$ Planet composition

\section{Introduction}

The conditions that a planet must fulfill to be habitable are not precisely known. It is comparatively easier to define conditions under which a planet is very likely not habitable.

It is known, at least in the case of the Earth, but also likely for many planets, that the operation of the carbon cycle (see for example Kasting 2010; Pierrehumbert 2010) is essential for habitability. Indeed, on Earth, the carbon cycle acts as a very important temperature stabilizing process and buffers the surface temperature at values close to those allowing liquid water. This is especially important since the luminosity of a star increases with time and, without any stabilization process, it could be difficult to maintain liquid water at a surface of any planet over an appreciable amount of time.

The stabilizing effect of the carbon cycle comes from both the dependence of silicate weathering on temperature and the strong greenhouse warming effect of $\mathrm{CO}_{2}$. Silicate weathering requires in turn a reaction between $\mathrm{CO}_{2}$ dissolved in the oceans, or directly with

Paper presented at the 2nd Annual Earth-Life Science Institute International Symposium, held at the National Institute of Informatics in Chiyoda-ku, Tokyo, from 24 to 26 March, 2014.

Yann Alibert

yann.alibert@space.unibe.ch

1 Center for Space and Habitability, Physikalisches Institut, Universität Bern, Bern, Switzerland 
$\mathrm{CO}_{2}$ present in the atmosphere, and rocks from the planetary mantle. This, in turn, is only possible if there is a physical interface between liquid water (or the atmosphere) and rocks.

Water-rich planets (or ocean planets) are covered by a global ocean. If the amount of water is large enough, the pressure at the bottom of the global ocean is so large that a layer of highpressure ice (ice VII) appears. This effectively prevents any contact between silicates and liquid water (and therefore atmospheric $\mathrm{CO}_{2}$ that could be dissolved in the ocean), and suppresses silicate weathering. As a result, the carbon cycle and its stabilizing effect cannot exist. As a consequence, planets with high enough water content, and therefore a high-pressure ice layer at the bottom of a global ocean, are not habitable.

For a given planetary mass, a large radius implies either the presence of a lot of water, or the presence of a massive gaseous envelope, or both. In the first case, habitability is hindered by the presence of a high-pressure ice layer at the bottom of the global ocean. In the second case, the temperature and pressure at the bottom of the atmosphere are too large to allow for the presence of liquid water. In both cases, the planet is therefore likely not habitable. Computing the maximum radius a planet can have in order not to be non-habitable is the goal of this paper.

For this, we need to compute, for any planetary mass, all the possible planetary structures compatible with a given bulk composition (see below). In the ensemble of these structures, we then select the ones that have no ice layer at the bottom of the ocean, and surface conditions compatible with the presence of liquid water. The maximum radius of all these structures is then the maximum radius a planet of the considered mass can have in order not to be nonhabitable. All planets larger (in term of radius) than this limit are not habitable.

\section{Methods}

We compute the internal structure of planets that consist of five layers:

- a core

- an inner mantle

- an outer mantle

- a water layer

- a gas envelope

The presence of a core depends on the assumed Fe/Si ratio in the planet. Indeed, for a given composition, the largest radius is obtained when the maximum amount of Fe is present in silicates. For each planetary composition, we therefore compute the structure with the smallest possible core (since we want to derive an upper boundary of the planetary radius). It appears that, for solar $\mathrm{Mg} / \mathrm{Si}$ and $\mathrm{Fe} / \mathrm{Si}$ ratios, all the available Fe can be present in the inner and outer mantle, and no core is required to match the composition. For Fe/Si $>1.13 \mathrm{Fe} / \mathrm{Si}_{\odot}$ (for the chemical species we consider in the model), this is no longer possible and planets have an iron core.

Our model of the four (three if no core is required) innermost layers follows closely the one proposed by Sotin et al. (2007) and further improved by Grasset et al. (2010). The core is made of Fe (for simplicity, we do not include any inner/outer core dichotomy and do not include the effect of the presence of volatiles such as $\mathrm{S}$ in the core), the inner mantle is made of perovskite $\left(\mathrm{MgSiO}_{3} / \mathrm{FeSiO}_{3}\right)$ and wustite $(\mathrm{MgO} / \mathrm{FeO})$, the outer mantle is made of olivine $\left(\mathrm{Mg}_{2} \mathrm{SiO}_{4} /\right.$ $\left.\mathrm{Fe}_{2} \mathrm{SiO}_{4}\right)$ and entstatite $\left(\mathrm{Mg}_{2} \mathrm{Si}_{2} \mathrm{O}_{6} / \mathrm{Fe}_{2} \mathrm{Si}_{2} \mathrm{O}_{6}\right)$. The water layer is assumed to be made of pure water, and the planetary envelope is assumed to be made of either $\mathrm{H}_{2}$ or $\mathrm{H} / \mathrm{He}$ in solar 
composition, the latter two being treated as ideal gases. This choice of the planetary atmospheric composition is dictated by our goal to derive a maximum planetary envelope: other choices of atmospheric composition (e.g., $\mathrm{O}_{2}, \mathrm{CO}_{2}, \mathrm{~N}_{2} \ldots$ ) would produce smaller radii.

The temperature profile in the different layers is assumed to be adiabatic, and the boundary conditions are as follows: we specify a surface temperature and pressure (in the range that allows the presence of liquid water), as well as a central pressure. In addition, we assume that the pressure at the bottom of the ocean is the equilibrium pressure, at the ice VII/water transition. The thermal profile in the outer mantle is then computed, following an adiabat, until the transition to the inner mantle, at a pressure of the order of $22 \mathrm{GPa}$. Again, this transition depends on the temperature, following a similar law as for the ice/water transition (see Alibert 2014). Finally, the temperature profile in the inner mantle and the core is computed following an adiabat, until the assumed central pressure is reached.

Once the thermal profile has been constructed, we integrate the internal structure equations, using the pressure as an independent variable. The only unknown in the model is therefore the pressure at the core/inner mantle interface (if there is enough Fe to allow the presence of an iron core). We therefore use an iterative method to find the transition pressure that allows matching the assumed composition of the planet (the $\mathrm{Fe} / \mathrm{Si}$ ratio in particular). Once the internal structure of the non-gaseous part of the planet has been derived, we compute the structure of the atmosphere by following an adiabat starting from the surface temperature and pressure, until the skin temperature is reached, following the method of Pierrehumbert and Gaidos (2011). The structure of the atmosphere is then used to derive the chord radius, corresponding to the place where the chord optical depth (the one that is observed by transit) is equal to 1 .

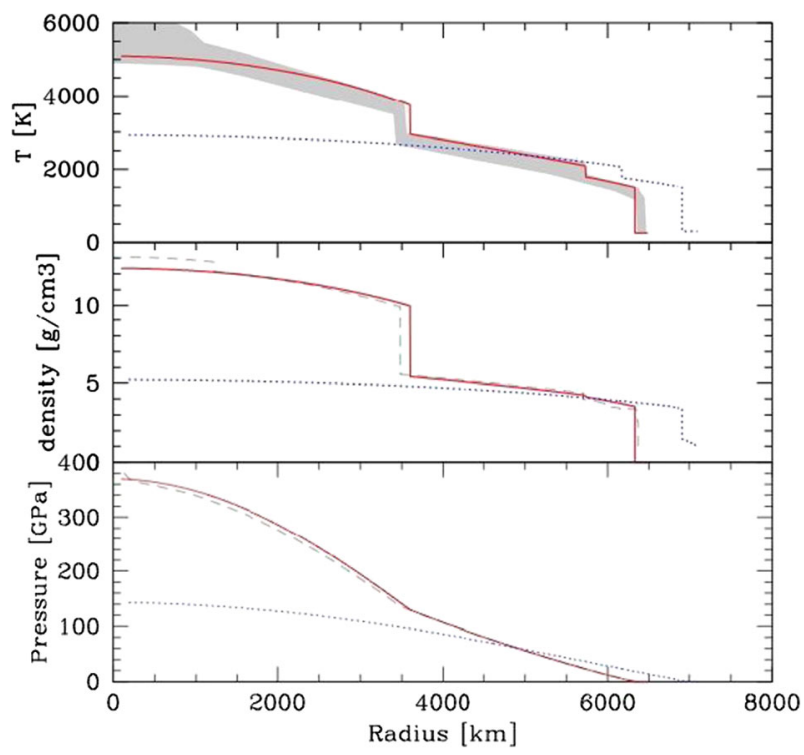

Fig. 1 Temperature, density and pressure (top, middle, bottom panels) as a function of radius for two models. Solid red lines: $1 M_{\oplus}$ planet with $T_{\text {surf }}=300 \mathrm{~K}, P_{\text {surf }}=1$ bar, without ocean, and quasi-solar composition (see text). Dotted blue lines: the same planet, but corresponding to the overall maximum models (iron free, inner mantle made of $\mathrm{MgO}$, outer mantle made of $\mathrm{Mg}_{2} \mathrm{Si}_{2} \mathrm{O}_{6}$, with maximum depth ocean). On the middle and bottom panels, the black dashed lines give the Preliminary Reference Earth Model of Dziewonski and Anderson (1981). Note that no temperature is given in this model. Instead, the grey area reproduces the likely temperature of Earth as mentioned in Sotin et al. (2007) 
As an example and test of our code, we present in Fig. 1 the temperature, pressure and mass as a function of the radius, for an Earth like planet. The surface conditions are $\mathrm{T}=300 \mathrm{~K}$ and $\mathrm{P}=1$ bar. The composition of the planet is similar to the one in Sotin et al. (2007), model 1, namely $\mathrm{Mg} / \mathrm{Si}=1.131$ and $\mathrm{Fe} / \mathrm{Si}=0.986$. We moreover assume, for the sake of comparison, than $13 \%$ of the core is made of FeS (treated using the Mie-Gruneisen-Debye equation of state (EOS)), and $87 \%$ is made of pure Fe. We refer the reader to Sotin et al. (2007) for the justification of this slightly non-solar composition. In order to have a planet of $1 M_{\oplus}$, the central pressure is equal to $370 \mathrm{GPa}$, the pressure at the core-mantle transition is equal to $130 \mathrm{GPa}$, and the pressure at the inner/outer mantle transition is equal to $23.31 \mathrm{GPa}$. The corresponding temperatures are respectively 5092, 3747 and $1789 \mathrm{~K}$. The mass obtained using these parameters is $1.007 M_{\oplus}$, and the radius of the solid part is $0.993 R_{\oplus}$. The pressure, density and temperature as a function of the radius are moreover similar to the Preliminary Reference Earth Model (Dziewonski and Anderson 1981), except for the inner/outer core structure that is not modeled in our code. This difference in the core structure, and mean density, also explains the slight shift in radius of the core-mantle boundary. For this type of planet, the maximum depth of the ocean, which is not taken into account in the numbers shown above, would be $150 \mathrm{~km}$, corresponding to a mass of $0.016 M_{\oplus}$, more than 100 times the inventory of water on Earth.

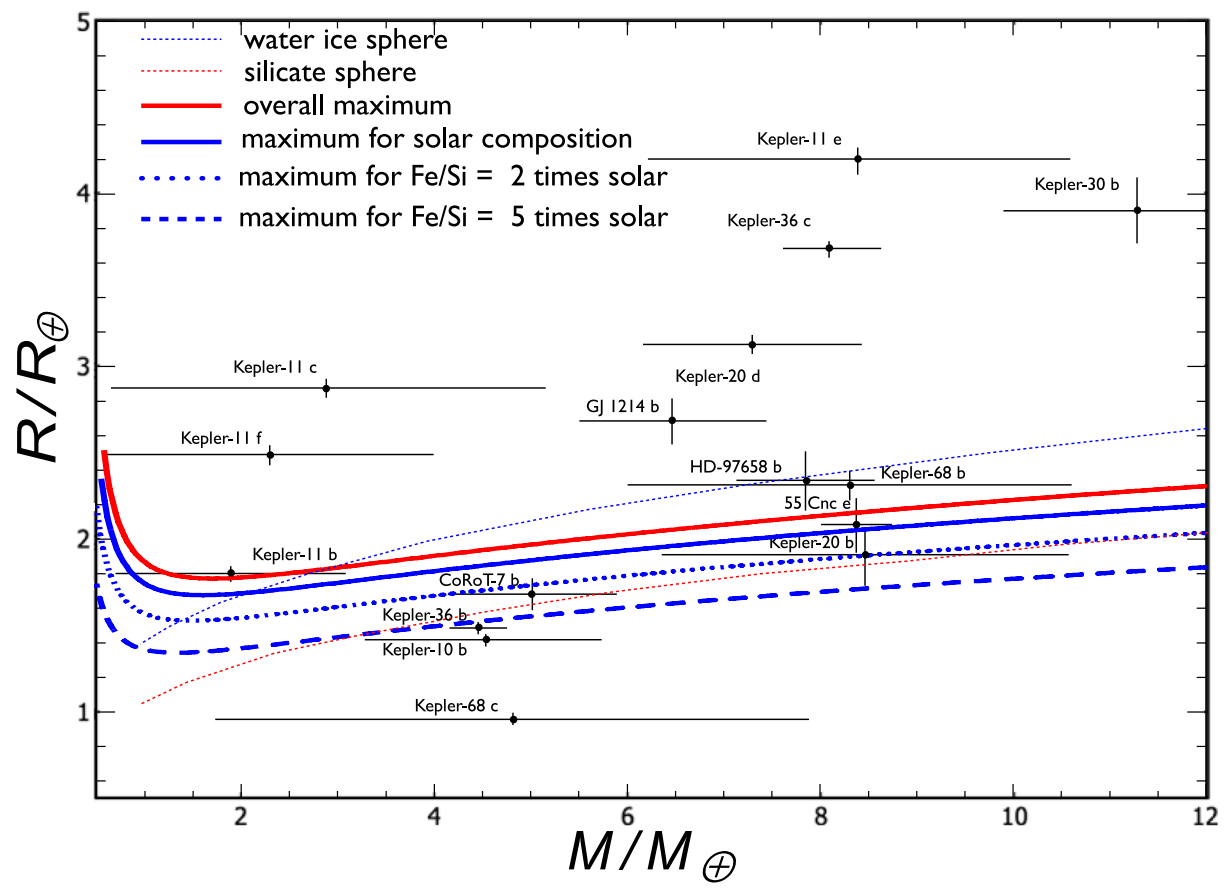

Fig. 2 Mass versus maximum radius relationship for different composition of the planetary interior. The heavy red solid line is in the case of an iron free planet, with an inner mantle made of $\mathrm{MgO}$, outer mantle made of $\mathrm{Mg}_{2} \mathrm{Si}_{2} \mathrm{O}_{6}$. The blue lines are computed assuming solar $\mathrm{Mg} / \mathrm{Si}$ and $\mathrm{Fe} / \mathrm{Si}$ ratios equal to the solar value (solid line), two times the solar value (dotted line), and five times the solar value (dashed line). The mass-radius relationships for a sphere of silicates and a sphere of water are indicated by thin solid lines (respectively red and blue), and are taken from Wagner et al. (2011). The parameters of some transiting planets are taken from exoplanets.org 


\section{Results}

Using the model described above, we now derive the maximum radius a planet can have in order to harbor both a carbon cycle and surface conditions in the range defined above. The overall maximum is obtained assuming that the planet has a very peculiar composition, where only low-density minerals are present in the planetary interior, namely $\mathrm{MgO}$ for the inner mantle, and $\mathrm{Mg}$-entstatite for the outer mantle. Figure 2 shows the resulting mass-radius relation we obtain, with some transiting planets also plotted on the figure (the data were taken from exoplanet.org). As can be seen, the maximum radius increases from $1.8 R_{\oplus}$ to $2.3 R_{\oplus}$ for planets ranging from 1 to $10 M_{\oplus}$. Lower mass planets can have a larger radius, the increase of the massradius relationship being the result of the reduced gravity. Such a behavior of the mass-radius relation has already been described in other studies (see Mordasini et al. (2012) and references therein). As can be seen in the figure, a large fraction of the planets represented on the figure are not habitable, based on the criteria described in the introduction (it is in nay event likely that these planets are not habitable, due to their proximity to their central star).

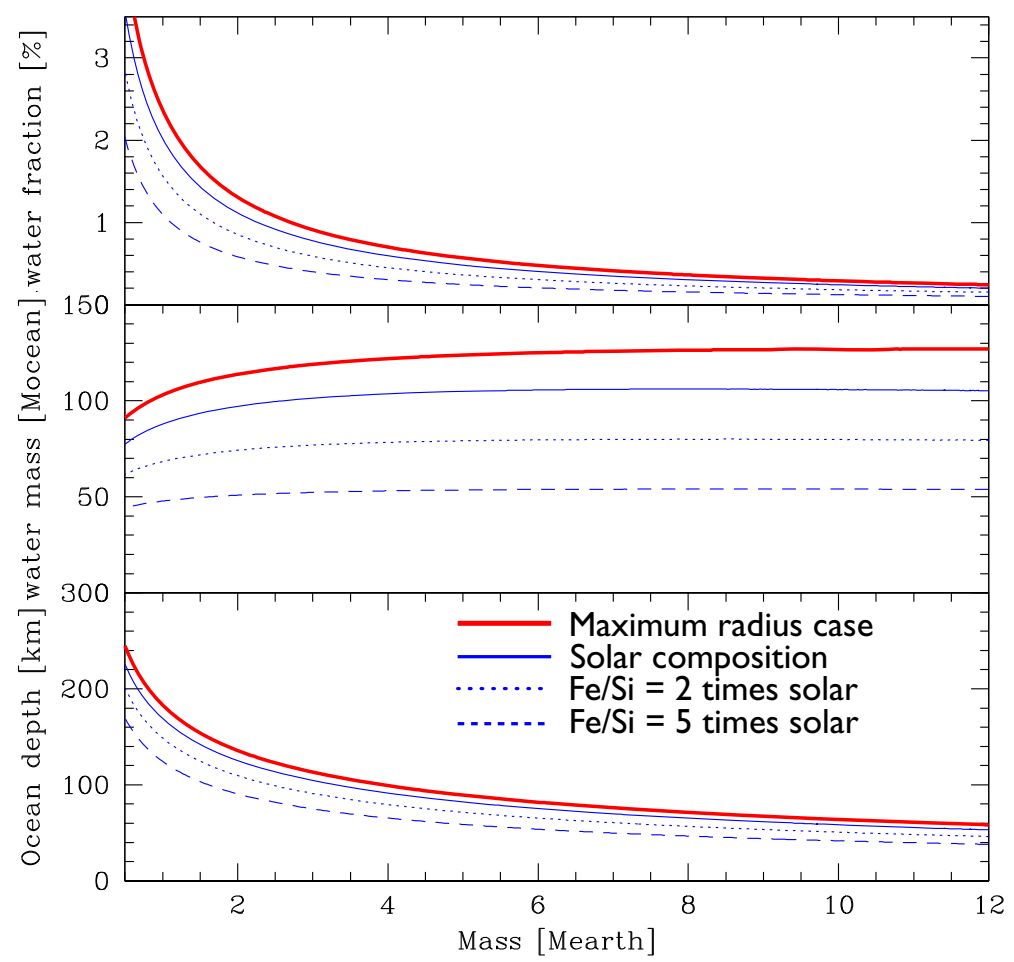

Fig. 3 Characteristics of the maximum ocean as a function of the planetary mass, for different models. The top, middle and bottom panels present respectively the water fraction (considering only the ocean), the ocean mass relative to the ocean mass on Earth $\left(M_{\text {Ocean }}=2.310^{-4} M_{\oplus}\right)$, and the ocean depth. The heavy red solid line is in the case of an iron-free planet, with an inner mantle made of $\mathrm{MgO}$ and an outer mantle made of $\mathrm{Mg}_{2} \mathrm{Si}_{2} \mathrm{O}_{6}$. The blue lines are computed assuming a solar $\mathrm{Mg} / \mathrm{Si}$ ratio, and a $\mathrm{Fe} / \mathrm{Si}$ ratio equal to the solar value (solid line), two times the solar value (dotted line), and five times the solar value (dashed line) 
It is interesting to also consider the maximum mass of the ocean on such planets. Figure 3 shows the maximum ocean mass, and the maximum water fraction as a function of the planetary mass. Note that the maximum water fraction is based on the ocean mass only, and does not include water that could be in reality incorporated in the planetary interior. The maximum water mass is found to be nearly independent of the planetary mass, except for low mass planets. Accordingly, the maximum water fraction decreases with planetary mass, from $2 \%$ for an Earth-mass planet to $0.2 \%$ for a $12 M_{\oplus}$ planet.

Assuming some values of the $\mathrm{Fe} / \mathrm{Si}$ and $\mathrm{Mg} / \mathrm{Si}$ ratios in the planetary interior (these values reflect approximately the similar ratios in the central star, see Thiabaud et al. (2014), and Marboeuf et al. (2014a, b)), we can repeat the same calculations. For these, the fraction of the different compounds (Fe, perovskite, wustite, olivine, entsatite) is computed assuming that the mantle is homogeneous (the $\mathrm{Mg} / \mathrm{Si}$ and $\mathrm{Fe} / \mathrm{Si}$ ratios are the same in the inner and outer mantle).

Figure 2 shows the resulting mass-radius relation for different values of the $\mathrm{Fe} / \mathrm{Si}$ ratio, namely 1,2 and 5 times the solar value (0.8511). The $\mathrm{Mg} / \mathrm{Si}$ ratio is kept at its solar value (1.0243) for all the simulations. As can be seen, the planets with solar composition have a maximum radius less than $0.1 R_{\oplus}$ smaller than the overall maximum radius derived in the previous section. The shift in radius is approximately independent of the planetary mass, but depends on the Fe/Si ratio: planets are around $0.2 R_{\oplus}$ smaller than the overall maximum for $\mathrm{Fe} /$ $\mathrm{Si}=2 \mathrm{Fe} / \mathrm{Si} i_{\odot}$, and $0.4 R_{\oplus}$ smaller for $\mathrm{Fe} / \mathrm{Si}=5 \mathrm{Fe} / \mathrm{Si}_{\odot}$. Correspondingly, the maximum mass of water decreases from $\sim 125 M_{\text {Ocean }}$ to 105,80 and $55 M_{\text {Ocean }}$ for Fe/Si ratios equal to 1,2 and 5 times the solar value, respectively (see Fig. 3).

\section{Conclusions}

We have derived the maximum radius of planets in the Earth to Super-Earth regime, according to the hypothesis that both the surface conditions at the planetary surface lie in the liquid domain of the water phase diagram, and that the pressure at the bottom of a putative global ocean is smaller than the liquid water/ice VII transition (approximately $2.4 \mathrm{GPa}$ ). As we have argued in the introduction, if these two conditions are indeed necessary for habitability, the maximum radius we derive delimits a region in term of radius above which planets are very likely non habitable.

Furthermore, it is possible to derive a stronger constraint on the maximum radius of a planet, if one can determine both the abundance of key elements in the central star (in particular $\mathrm{Fe}, \mathrm{Si}$ and $\mathrm{Mg}$ ), and the mean molecular weight of the gas envelope, using Rayleigh scattering observations (see Benekke and Seager (2012)). Such a derivation relies however on the assumption that the refractory composition of planets is similar to the one of the central star, an assumption that is supported by recent population synthesis models (Thiabaud et al. (2014)). Using such models, transit observations like the one that will be performed by CHEOPS (Broeg et al. 2013) or TESS (Ricker et al. 2010), complimented by ground-based or other spacebased observations, will make it possible to select the best candidates for future habitability studies.

Acknowledgments This work was supported by the European Research Council under grant 239605 


\section{References}

Alibert Y (2014) Astron Astrophys 561:41

Benekke B, Seager S (2012) Ap J 753:100

Broeg C et al (2013) EPJW 47:3005

Dziewonski AM, Anderson DL (1981) Phys Earth Planet Inter 25:297

Grasset O, Schneider J, Sotin C (2010) ApJ 693:722

Kasting J (2010) How to find a habitable planet. Princeton University Press, Princeton

Marboeuf U, Thiabaud A., Alibert Y, Cabral N, Benz W (2014a) Adv Astron Astrophys 570, A36

Marboeuf U, Thiabaud A., Alibert Y, Cabral N, Benz W (2014b) Adv Astron Astrophys 570, A35

Mordasini C, Alibert Y, Klahr H, Henning T (2012) Astron Astrophys 547:111

Pierrehumbert R (2010) Principles of planetary climate. Cambridge University Press, Cambridge

Pierrehumbert R, Gaidos E (2011) ApJ 734:L13

Ricker GR et al (2010) AAS Meeting 215:450.06

Sotin C, Grasset O, Mocquet A (2007) Icarus 191:337

Thiabaud A et al (2014) Astron Astrophys 562:27

Wagner FW et al (2011) Icarus 214:366 